\title{
Subclinical hypothyroidism is associated with basal ganglia enlarged perivascular spaces and overall cerebral small vessel disease load
}

\author{
Ming Chu" ${ }^{1 \#}$, Yinyuan $\mathrm{Cai}^{2 \#}$, Jie Zhong ${ }^{3 \#}$, Yun Qian ${ }^{2}$, Yan $\mathrm{Cen}^{2}$, Miaomiao Dou ${ }^{2}$, Guilin Chen ${ }^{4}$, Bo Sun , \\ Xiaowei $\mathrm{Lu}^{2}$ \\ ${ }^{1}$ Department of Cardiology, The First Affiliated Hospital of Nanjing Medical University, Nanjing, China; ${ }^{2}$ Department of Geriatrics, The First \\ Affiliated Hospital of Nanjing Medical University, Nanjing, China; ${ }^{3}$ School of Foreign Languages, Nanjing University of Finance \& Economics, \\ Nanjing, China; ${ }^{4}$ Department of Radiology, The First Affiliated Hospital of Nanjing Medical University, Nanjing, China; ${ }^{5}$ Department of Neurology, \\ The Affiliated Huaian No. 1 People's Hospital of Nanjing Medical University, Huai'an, China
}

Contributions: (I) Conception and design: X Lu, M Chu, B Sun; (II) Administrative support: X Lu; (III) Provision of study materials or patients: Y Cai, Y Cen, M Dou, G Chen; (IV) Collection and assembly of data: Y Qian, Y Cai, Y Cen, M Dou; (V) Data analysis and interpretation: X Lu, M Chu, J Zhong; (VI) Manuscript writing: All authors; (VII) Final approval of manuscript: All authors.

"These authors contributed equally to this work.

Correspondence to: Xiaowei Lu, MD. Department of Geriatrics, The First Affiliated Hospital of Nanjing Medical University, Guangzhou Road 300\#, Nanjing 210029, China. Email: njluxiaowei@163.com.

Background: The association between subclinical hypothyroidism (SCH) and cerebral small vessel disease (CSVD) in the stroke-free population is currently unclear.

Methods: A total of 354 individuals without a history of stroke were enrolled in this study. Demographic data, medical history, vascular risk factors, carotid arteriosclerosis, and the results of laboratory tests were collated. SCH is defined as an elevation in thyroid-stimulating hormone levels, but with normal free thyroxine levels. Magnetic resonance imaging (MRI) was used to assess 4 markers of CSVD, including white matter hyperintensities (WMHs), lacunes, deep microbleeds, and enlarged perivascular spaces (EPVSs). The overall CSVD load was then ranked using an ordinal scale ranging from 0 to 4 . Brain atrophy was measured semiquantitatively on MRI. A binary logistic regression model was used to explore the association of SCH with each CSVD marker after adjusting for confounding factors. The ordinal regression model was used to explore the association of SCH with CSVD burden and brain atrophy after adjusting for confounding factors.

Results: The mean age of the participants (66.9\% males) was $69.4 \pm 12.8$ years. SCH was observed in 44 (12.4\%) participants. MRI findings revealed $13 \%$ of cases with lacunes, $6.2 \%$ with microbleeds, $50.3 \%$ with confluent WMH, and 49.2\% with extensive basal ganglia EPVS. Assessment of total CSVD burden showed that $29.1 \%$ scored $1,30.5 \%$ scored 2, 6.5\% scored 3, and 2.3\% scored $\geq 3$. SCH was associated with extensive basal ganglia EPVS [odds ratio $(\mathrm{OR})=2.175 ; 95 \%$ confidence interval (CI): 1.075 to 4.401 ] and total CSVD load (OR $=1.879 ; 95 \% \mathrm{CI}: 1.028$ to 3.438$)$. $\mathrm{SCH}$ was not associated with advanced brain atrophy.

Conclusions: SCH is associated with the advanced total burden of CSVD and basal ganglia EPVS in the stroke-free population.

Keywords: Subclinical hypothyroidism (SCH); cerebral small vessel disease (CSVD); stroke-free

Submitted Feb 15, 2021. Accepted for publication Aug 27, 2021.

doi: 10.21037/qims-21-190

View this article at: https://dx.doi.org/10.21037/qims-21-190 


\section{Introduction}

Cerebral small vessel disease (CSVD) refers to abnormal pathologies affecting the brain's small arteries, arterioles, venules, and capillaries (1). It is associated with a significant risk of cognitive impairment, stroke, gait disturbances, and other poor outcomes (1). Clinically, magnetic resonance imaging (MRI) is used to detect biomarkers of CSVD, including white matter hyperintensities $(\mathrm{WMH})$, lacunes, and deep cerebral microbleeds (CMBs) $(2,3)$. Increasingly, other markers, such as enlarged perivascular spaces (EPVS) and brain atrophy, have been suggested $(1,4)$. Unfortunately, the precise etiology of CSVD is still not fully understood.

Studies have reported that arteriolosclerosis and artery and vascular endothelium dysfunction may contribute to CSVD (1-4). Recent investigations have suggested that subclinical hypothyroidism may cause arteriosclerosis and vascular endothelium dysfunction $(5,6)$ and may have a pathological role in CSVD $(1,4)$. Subclinical hypothyroidism is defined as elevated thyroid-stimulating hormone (TSH) with normal thyroid hormone levels (7) and may affect 4-20\% of the adult population $(7,8)$. Subclinical hypothyroidism has been reported to be associated with WMH, lacunes, and microbleeds, but its relationship with EPVS is still unclear $(9,10)$. For instance, in patients suffering from acute stroke, no relationship between subclinical hypothyroidism and EPVS was found $(9,10)$. However, since thyroid function changes with the recovery from strokes, the relationship should be reassessed after recovery $(11,12)$.

In this study, the relationship between subclinical hypothyroidism and the risk of individual CSVD lesions (assessed using the biomarkers WMH, lacunes, microbleeds, and EPVS), the overall CSVD load, and brain atrophy in the stroke-free population was assessed.

\section{Methods}

\section{Study participants}

Stroke-free individuals who underwent a health checkup in the First Affiliated Hospital with Nanjing Medical University from July 2016 to December 2018, were consecutively and retrospectively screened for eligibility to this study. Patients were included if they satisfied the following inclusion criteria: (I) no history of stroke; (II) $\mathrm{TSH}$, free thyroxine (FT4), and free triiodothyronine (FT3) data were available (thyroid-related antibodies were not routinely tested); (III) complete echocardiography data were available (Philipa, Netherland); (IV) assessment of carotid arteriosclerosis was conducted by Doppler Ultrasound (Toshiba, Japan); and (V) MRI data were available. Patients were excluded if they presented with the following: (I) overt thyroid disease defined by TSH $>10 \mathrm{mIU} / \mathrm{L}, \mathrm{FT} 4$ out of reference range, or FT3 out of reference range; (II) a history of thyroid diseases, or were treated with antithyroid medications or replacement thyroxine therapy; (III) bright signal on diffusion-weighted imaging (DWI)-MRI; (IV) carotid arteriosclerosis with lumen stenosis greater than $70 \%$ and left ventricular ejection fraction $<50 \%$; and $(\mathrm{V})$ a history of brain tumor, brain trauma, or malignancy.

Basic patient demographic data (age and gender), medical history, vascular risk factors, and the serum concentrations of total cholesterol, low-density lipoprotein cholesterol, and creatinine [for the calculation of estimated glomerular filtration rate by Chronic Kidney Disease Epidemiology Collaboration (CKD-EPI) formula] were collated.

This study was conducted in accordance with the Declaration of Helsinki (as revised in 2013). The study protocols were approved by the Ethics Committee of the First Affiliated Hospital with Nanjing Medical University, and informed consent was obtained from all subjects or their representatives.

\section{Carotid artery ultrasonography}

Ultrasonography was used to study the carotid artery with a machine (Toshiba, Japan) equipped with a $7.5-\mathrm{MHz}$ transducer. The measurement of bilateral carotid arteries was performed at the level of the common carotid, the bifurcation, and the internal carotid arteries in transverse and longitudinal orientations. Experienced sonographers blinded to the clinical and laboratory data were responsible for measuring intima-media thickness (IMT). An isolated focal thickening of the intima-media layer with a thickness of $\geq 1.3 \mathrm{~mm}$ was defined as a characteristic of arteriosclerosis. Arteriosclerosis was measured at the common or internal carotid artery or the carotid bulb. Carotid arteriosclerosis was defined as IMT $>10 \mathrm{~mm}$ or the presence of arteriosclerosis.

\section{Thyroid function tests}

Peripheral serum was collected to determine the concentrations of serum TSH, FT4, and FT3 using an automated electrochemiluminescence immunoassay (IMMULITE, Diagnostic Products Corporation, CA, USA). According to the international standard (7), elevated TSH levels (4.5-10.0 mIU/L) with normal FT4 levels 


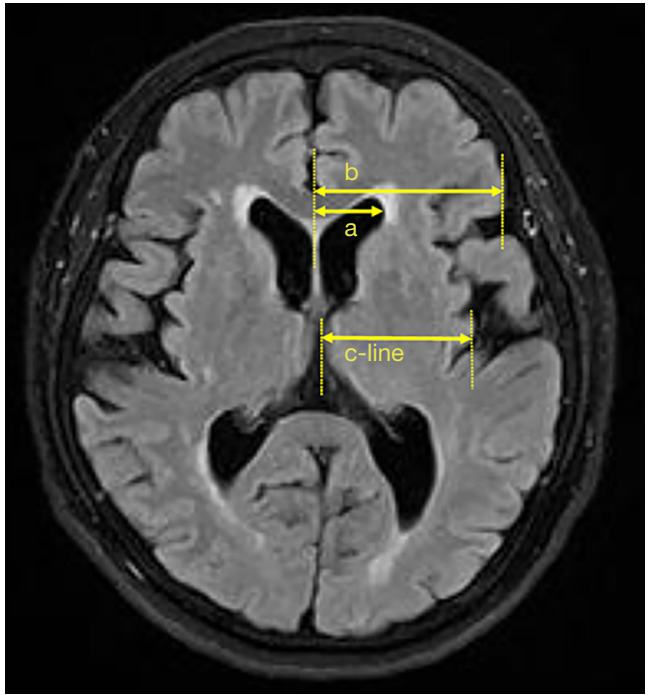

Figure 1 Magnetic resonance imaging (MRI) was used for the semi-quantitative assessment of brain atrophy. The $a / b$ ratio and the measurement of c-line was calculated, where "a" is defined as the maximum measurement from the central line to the lateral margin of the anterior horn of the lateral ventricle and " $b$ " is defined as the maximum measurement from the central line to the lateral margin of the frontal lobe at the axial level of the basal ganglia. The "c-line" is defined as the maximum measurement from the central line to the lateral margin of insular cortex at the same level.

(9.0-24.4 $\mathrm{pmol} / \mathrm{L})$ were defined as subclinical hypothyroidism. Individuals with normal thyroid function were assigned to the normal group. The normal reference range for TSH, FT4, and FT3 are 0.41-4.49 mIU/L, 9.0-24.4 pmol/L, and 3.1-6.6 pmol/L, respectively.

\section{Neuroimaging protocol and analysis}

Brain MRI (3.0 Tesla, TIM Trio, Simens, Erlangen, Germany) was used to assess CSVD within 7 days after admission. All patients received T1-weighted imaging, T2-weighted imaging (T2WI), fluid-attenuated inversion recovery (FLAIR), DWI, and T2*-weighted gradient echo images (T2*-GE) using standard protocols (2). Confluent deep WMH or irregular periventricular hyperintensity, which extends into the deep white matter on T2WI and FLAIR, was defined as confluent WMH. Silent lacunar infarction was defined as fluid-filled cavities, with a diameter $<20 \mathrm{~mm}$, located in the territory of a perforating arteriole. T2*-GE was used to detect deep CMBs both in white matter and basal ganglia. EPVS was characterized as small, sharply delineated structures of cerebrospinal fluid intensity on imaging that followed the orientation of the perforating vessels and ran perpendicular to the brain surface. High signals indicated the EPVS on T2 and low signals on T1, and FLAIR sequences. The EPVS was unilaterally counted at the axial level of the basal ganglia, and the side with more EPVS was selected. All ratings were independently performed by two experienced neurologists blinded to the patients' clinical data. Any disagreements between the neurologists were resolved via discussion. To measure total CSVD burden, an ordinal scoring scale with a minimum of 0 and a maximum of 4 was constructed. One point was awarded for the presence of each of the following items: confluent WMH; $\geq 1$ silent lacunar infarctions; $\geq 1$ deep CMBs; $>10$ basal ganglia EPVS. Brain atrophy was semi-quantitatively indicated by the ratio of $\mathrm{a} / \mathrm{b}$ and the measurement of c-line on MRIs as previously reported, with minor modifications (13), where "a" is defined as the maximum measurement from the central line to the lateral margin of the anterior horn of the lateral ventricle and " $b$ " is the maximum measurement from the central line to the lateral margin of frontal lobe at the axial level of the basal ganglia. The "c-line" is defined as the maximum measurement from the central line to the lateral margin of the insular cortex at the same level (Figure 1).

\section{Statistical analysis}

The SPSS software (version 22.0) was used for all statistical analyses. Categorical variables are expressed as $\mathrm{n}(\%)$, and continuous variables are expressed as mean \pm standard deviation (SD). Differences in baseline characteristics between two groups were analyzed using $\chi^{2}$ or KruskalWallis test where appropriate. Binary logistic regression was used to explore the association of subclinical hypothyroidism with individual CSVD markers, including confluent WMH, lacuna, microbleeds, and EPVS. Ordinal regression was performed to investigate the association of subclinical hypothyroidism with the overall CSVD load and brain atrophy (increase in the $\mathrm{a} / \mathrm{b}$ ratio or reduction of the c-line distance, where $\mathrm{a} / \mathrm{b}$ and $\mathrm{c}$-line distance were categorized as quartiles). Statistical significance was defined as $\mathrm{P}<0.05$ (two-tail).

\section{Results}

\section{Demographic, laboratory, and clinical parameters}

A total of 354 participants $(66.9 \%$ males) with a mean 
Table 1 The demographic, laboratory, and clinical parameters of the participants grouped by subclinical hypothyroidism (SCH)

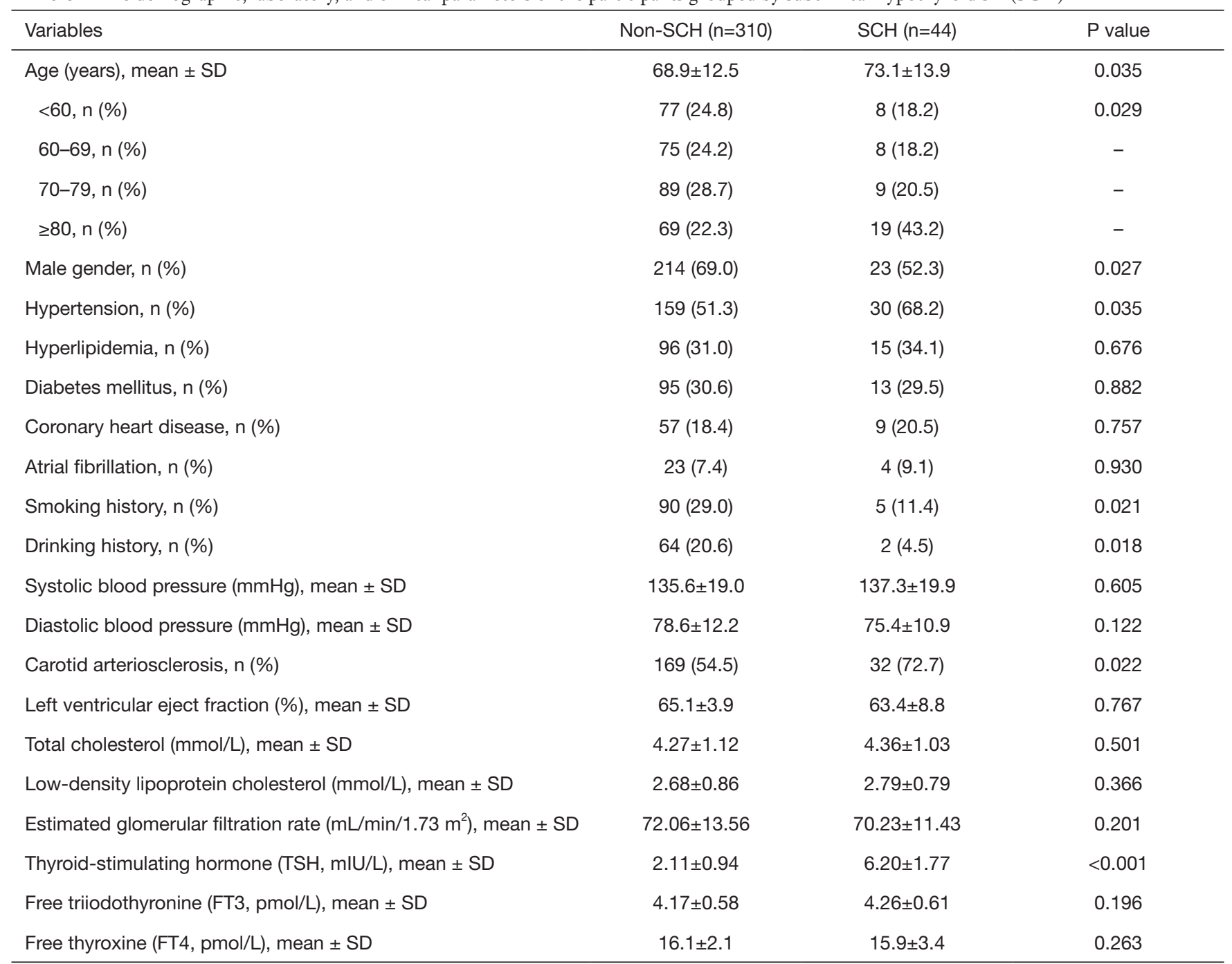

$\mathrm{SD}$, standard deviation.

age of $69.4 \pm 12.8$ years was enrolled in this study. In this cohort, $53.4 \%$ presented with hypertension, $31.4 \%$ had hyperlipidemia, $30.5 \%$ had diabetes mellitus, $7.6 \%$ had atrial fibrillation, $56.8 \%$ had carotid arteriosclerosis, and $18.6 \%$ presented with coronary heart disease. A history of smoking was reported in $26.8 \%$ of participants, and $18.6 \%$ reported a history of drinking. Subclinical hypothyroidism was observed in $44(12.4 \%)$ patients. Table 1 illustrates the demographic, laboratory, and clinical characteristics of the participants grouped by subclinical hypothyroidism. Patients with subclinical hypothyroidism tended to be older $(\mathrm{P}=0.035)$, male $(\mathrm{P}=0.027)$, experienced more prevalent hypertension $(\mathrm{P}=0.035)$ and carotid arteriosclerosis $(\mathrm{P}=0.022)$, and were less likely to have a history of smoking
$(\mathrm{P}=0.021)$ and drinking $(\mathrm{P}=0.018)$.

\section{Individual CSVD markers and overall CSVD load on MRI}

Based on MRI results (Table 2), 112 patients (31.6\%) were not positive for any CSVD markers, whereas 8 patients (2.3\%) were positive for all 4 markers of CSVD. For individual CSVD markers, 178 patients (50.3\%) presented with confluent WMH, 174 cases $(49.2 \%)$ had EPVS, 46 cases (13.0\%) had lacuna, and 22 cases (6.2\%) had microbleeds. Most CSVD patients $(n=242)$ had 1 or 2 markers of CSVD (103/242, 42.6\% and 108/242, 44.6\%, respectively). Patients with subclinical hypothyroidism tended to have more prevalent EPVS 
Table 2 Magnetic resonance imaging (MRI) biomarkers of CSVD and brain atrophy in enrolled participants grouped by subclinical hypothyroidism $(\mathrm{SCH})$

\begin{tabular}{|c|c|c|c|}
\hline Variables & Non-SCH $(n=310)$ & $\mathrm{SCH}(\mathrm{n}=44)$ & $P$ value \\
\hline Confluent WMH, n (\%) & $152(49.0)$ & $26(59.1)$ & 0.212 \\
\hline Lacuna, n (\%) & $38(11.3)$ & $8(18.2)$ & 0.274 \\
\hline Basal ganglia-EPVS, n (\%) & $144(46.5)$ & $30(68.2)$ & 0.007 \\
\hline Total load of CSVD, n (\%) & & & 0.002 \\
\hline 0 & $107(34.5)$ & $5(11.4)$ & - \\
\hline 1 & $83(26.8)$ & $20(45.5)$ & - \\
\hline 2 & $97(31.3)$ & $11(25.0)$ & - \\
\hline \multicolumn{4}{|l|}{ Brain atrophy, mean \pm SD } \\
\hline$a / b$ & $0.325 \pm 0.039$ & $0.330 \pm 0.041$ & 0.555 \\
\hline c-line distance $(\mathrm{mm})$ & $20.4 \pm 6.0$ & $19.4 \pm 6.6$ & 0.099 \\
\hline
\end{tabular}

Notes: "a" is defined as the maximum measurement from the central line to the lateral margin of anterior horn of lateral ventricle; "b" is defined as the maximum measurement from the central line to the lateral margin of the frontal lobe at the axial level of the basal ganglia; "c-line" is defined as the maximum measurement from the central line to the lateral margin of the insular cortex at the same level. CSVD, cerebral small vessel diseases; WMH, white matter hyperintensities; EPVS, enlarged perivascular spaces; SD, standard deviation.

$(\mathrm{P}=0.007)$ and increased severity of overall CSVD load $(\mathrm{P}=0.002)$. However, the incidence of confluent $\mathrm{WMH}$ $(\mathrm{P}=0.212)$, lacuna $(\mathrm{P}=0.274)$, and microbleeds $(\mathrm{P}=0.609)$ were not significantly different between the subclinical hypothyroidism group and the normal group. In addition, neither the $\mathrm{a} / \mathrm{b}$ ratio $(\mathrm{P}=0.555)$ nor the $\mathrm{c}$-line distance $(\mathrm{P}=0.099)$ was significantly different between the 2 groups.

\section{The relationship between subclinical hypothyroidism and individual CSVD markers and brain atrophy}

Hypertension, hyperlipidemia, diabetes mellitus, smoking history, and drinking history are traditional vascular risk factors. The factors that showed significant differences were further analyzed, including age, gender, hypertension, smoking history, drinking history, and carotid arteriosclerosis. Coronary heart disease was also examined as a confounder because stroke is considered an isocritical condition of coronary heart disease.

As shown in Table 3, binary logistic regression analysis revealed a significant association between subclinical hypothyroidism and increased risk of EPVS [Model 1: odds ratio $(\mathrm{OR})=2.175,95 \%$ confidence interval $(\mathrm{CI}): 1.075$ to $4.401, \mathrm{P}=0.031$ ]. This association was still evident after controlling for confounding factors including age, gender, vascular risk factors, coronary heart disease, and carotid arteriosclerosis, as indicated by Model 2 (OR $=2.261,95 \%$ CI: 1.105 to $4.628, \mathrm{P}=0.026)$ and Model $3(\mathrm{OR}=2.085$, 95\% CI: 1.012 to $4.296, \mathrm{P}=0.046)$. However, no significant association was found between subclinical hypothyroidism and confluent $\mathrm{WMH}$, lacuna, nor microbleeds in any of the 3 models.

Classification by quartiles revealed that increases in the $\mathrm{a} / \mathrm{b}$ ratio and c-line distance reductions were associated with age, male gender, and EPVS. However, neither the increase of $\mathrm{a} / \mathrm{b}$ nor the reduction of c-line distance was associated with subclinical hypothyroidism (Table S1).

\section{The relationship between subclinical hypothyroidism and overall CSVD load}

In Table 4, ordinal regression analysis showed that advanced 
Table 3 The relationship between subclinical hypothyroidism and individual cerebral small vessel disease markers

\begin{tabular}{|c|c|c|c|c|c|c|c|c|c|}
\hline Variables & \multicolumn{3}{|c|}{ Model 1} & \multicolumn{3}{|c|}{ Model 2} & \multicolumn{3}{|c|}{ Model 3} \\
\hline Confluent WMH & 1.119 & $0.555-2.257$ & 0.753 & 1.372 & $0.649-2.899$ & 0.407 & 1.221 & $0.573-2.603$ & 0.605 \\
\hline Lacuna & 1.341 & $0.561-3.205$ & 0.509 & 1.626 & $0.641-4.125$ & 0.307 & 1.605 & $0.631-4.083$ & 0.320 \\
\hline Basal ganglia-EPVS & 2.175 & $1.075-4.401$ & 0.031 & 2.261 & $1.105-4.628$ & 0.026 & 2.085 & $1.012-4.296$ & 0.046 \\
\hline
\end{tabular}

For the binary logistic regression analysis, Model 1 was adjusted by age and gender; Model 2 was adjusted by age, gender, vascular risk factors, and coronary heart disease; Model 3 was adjusted by age, gender, vascular risk factors, coronary heart disease, and carotid arteriosclerosis. WMH, white matter hyperintensities; EPVS, enlarged perivascular spaces; OR, odds ratio; Cl, confidence interval.

Table 4 The relationship between subclinical hypothyroidism (SCH) and advanced total load of cerebral small vessel disease (CSVD)

\begin{tabular}{|c|c|c|c|c|}
\hline Variables & Unadjusted OR (95\% Cl) & $P$ value & Adjusted OR (95\% Cl) & $P$ value \\
\hline$<60$ & 1 (Referent) & & 1 (Referent) & \\
\hline $60-69$ & $2.221(1.237-3.991)$ & 0.008 & $2.173(1.191-3.959)$ & 0.011 \\
\hline $70-79$ & $4.437(2.472-7.957)$ & $<0.001$ & $4.504(2.460-8.239)$ & $<0.001$ \\
\hline Hypertension & $2.430(1.573-3.751)$ & $<0.001$ & $2.394(1.523-3.766)$ & $<0.001$ \\
\hline Carotid arteriosclerosis & $2.339(1.312-3.507)$ & 0.002 & $2.158(1.219-3.683)$ & 0.009 \\
\hline $\mathrm{SCH}$ & $1.879(1.028-3.438)$ & 0.040 & $1.848(1.004-3.401)$ & 0.048 \\
\hline
\end{tabular}

For ordinal regression analysis, all covariates included age, gender, vascular risk factors, coronary heart disease, carotid arteriosclerosis, and $\mathrm{SCH}$. OR, odds ratio; $\mathrm{Cl}$, confidence interval.

age, hypertension (OR $=2.430,95 \% \mathrm{CI}: 1.573$ to 3.751$)$, carotid arteriosclerosis (OR $=2.339,95 \%$ CI: 1.312 to 3.507 ), and subclinical hypothyroidism $(\mathrm{OR}=1.879,95 \% \mathrm{CI}: 1.028$ to 3.438) were significantly related to the higher total load of CSVD. These associations were still significant after controlling for vascular risk factors and coronary heart disease.

\section{Discussion}

This study demonstrated that subclinical hypothyroidism was significantly related to overall CSVD load and EPVS in the stroke-free population. Moreover, these relationships were still evident after controlling for possible confounding factors.

Several studies have revealed an independent association between subclinical hypothyroidism and the total burden of CSVD in patients of acute stroke $(9,10)$. The present study performed in stroke-free people also demonstrated a significant association between subclinical hypothyroidism and overall CSVD load. Reports regarding the relationship between subclinical hypothyroidism and individual CSVD markers are inconclusive. The Berlin "Cream \& Sugar" Study showed that TSH levels were independently associated with the severity of WMH (9). Another hospital-based study suggested a relationship between subclinical hypothyroidism and $\mathrm{WMH}$ and deep CMBs in patients with transient ischemia attack or minor stroke (10). This current study demonstrated an independent association between subclinical hypothyroidism and EPVS in a non-stroke population. It is possible that TSH levels may increase during the recovery of ischemic stroke, and this may affect the relationship between subclinical hypothyroidism and individual CSVD markers $(11,12)$. It has been reported that subclinical hypothyroidism is associated with diffuse loss of cortex and white matter in connected and unrelated regions (14) and eventually contributes to cognitive impairment (15). In the current study, the $\mathrm{a} / \mathrm{b}$ ratio and the c-line distance were used to indicate brain atrophy, according to previous 
studies (13). However, neither the $\mathrm{a} / \mathrm{b}$ ratio nor the $\mathrm{c}$-line distance was associated with subclinical hypothyroidism.

CSVD is a dynamic, whole-brain disorder that is commonly observed in the elderly population worldwide $(16,17)$. The underlying mechanisms of CSVD include arteriolosclerosis, artery stiffness, and vascular endothelium dysfunction $(1,2,4)$. While the relationship between subclinical hypothyroidism and CSVD is not fully understood, subclinical hypothyroidism has been reported to increase with age. It is associated with vascular resistance, arterial wall thickening, and endothelial dysfunction, which suggests that subclinical hypothyroidism may initiate the development of CSVD $(5,6,18)$. In support of this hypothesis, the current study demonstrated that participants with subclinical hypothyroidism experienced more prevalent hypertension and carotid arteriosclerosis.

An important function of the perivascular space is to drain interstitial fluid back to the circulation (19). The perivascular space is subject to blood-brain barrier dysfunction and increases in intra- and extra-cranial vascular pulsatility $(20,21)$. While most patients with subclinical hypothyroidism do not exhibit clinical symptoms, subclinical hypothyroidism can negatively affect the vascular system $(5,6)$. For instance, when the vessels are stiff, high pulsatile waves are transmitted to the vascular bed of the terminal organ, forcing the arterioles to adapt to higher blood flow, which might impair endothelial function and compromised the drainage of interstitial fluid $(4,22)$. Eventually, the perivascular spaces lose the ability to drain interstitial fluid and become enlarged (23).

The current study had several strengths. The strokefree status of patients was determined according to a positive history or positive findings on diffusion-weighted MRI. Both carotid arteriosclerosis and left ventricular eject fraction were assessed. Furthermore, a comprehensive assessment of various visible CSVD markers on brain MRI and several covariates were applied to analyze the relationship between subclinical hypothyroidism and CSVD. The relationship between subclinical hypothyroidism and brain atrophy was also investigated.

However, a causal relationship could not be established between subclinical hypothyroidism and CSVD, as there were several limitations to the current study. First, data were collected from a single center with a sample size of 354 , which may lead to sampling bias. Second, data were observational, and the location of lacuna and the volume of $\mathrm{WMH}$ were not measured. Third, this is a cross-sectional study, and single measurements of TSH, FT3, and FT4 may lead to inevitable misclassification. Future large-scale longitudinal studies with repeated measurements of TSH, FT3, and FT4 are warranted to address these issues.

\section{Conclusions}

The results in this report suggested that subclinical hypothyroidism is related to CSVD. The potential explanation for the association of subclinical hypothyroidism with CSVD may include microvascular brain damage and perivascular space dysfunction. The underlying pathophysiological mechanisms and therapeutic considerations require further clarification.

\section{Acknowledgments}

Funding: This work was supported by the National Natural Science Foundation of China (Grant No. 81771286).

\section{Footnote}

Conflicts of Interest: All authors have completed the ICMJE uniform disclosure form (available at https://dx.doi. org/10.21037/qims-21-190). The authors have no conflicts of interest to declare.

Ethical Statement: The authors are accountable for all aspects of the work in ensuring that questions related to the accuracy or integrity of any part of the work are appropriately investigated and resolved. The study was conducted in accordance with the Declaration of Helsinki (as revised in 2013). This study protocol was approved by the Ethics Committee of the First Affiliated Hospital with Nanjing Medical University, and informed consent was obtained from all subjects or their representatives.

Open Access Statement: This is an Open Access article distributed in accordance with the Creative Commons Attribution-NonCommercial-NoDerivs 4.0 International License (CC BY-NC-ND 4.0), which permits the noncommercial replication and distribution of the article with the strict proviso that no changes or edits are made and the original work is properly cited (including links to both the formal publication through the relevant DOI and the license). See: https://creativecommons.org/licenses/by-nc-nd/4.0/.

\section{References}

1. Wardlaw JM, Smith C, Dichgans M. Small vessel disease: 
mechanisms and clinical implications. Lancet Neurol 2019; 18:684-96.

2. Wardlaw JM, Smith EE, Biessels GJ, Cordonnier C, Fazekas F, Frayne R, et al. Neuroimaging standards for research into small vessel disease and its contribution to ageing and neurodegeneration. Lancet Neurol 2013;12:822-38.

3. Pantoni L. Cerebral small vessel disease: from pathogenesis and clinical characteristics to therapeutic challenges. Lancet Neurol 2010;9:689-701.

4. Riba-Llena I, Jiménez-Balado J, Castañé X, Girona A, López-Rueda A, Mundet X, Jarca CI, ÁlvarezSabin J, Montaner J, Delgado P. Arterial Stiffness Is Associated With Basal Ganglia Enlarged Perivascular Spaces and Cerebral Small Vessel Disease Load. Stroke 2018;49:1279-81.

5. Yao K, Zhao T, Zeng L, Yang J, Liu Y, He Q, Zou X. Non-invasive markers of cardiovascular risk in patients with subclinical hypothyroidism: A systematic review and meta-analysis of 27 case control studies. Sci Rep 2018;8:4579.

6. Nomoto S, Kinno R, Ochiai H, Kubota S, Mori Y, Futamura A, Sugimoto A, Kuroda T, Yano S, Murakami H, Shirasawa T, Yoshimoto T, Minoura A, Kokaze A, Ono $\mathrm{K}$. The relationship between thyroid function and cerebral blood flow in mild cognitive impairment and Alzheimer's disease. PLoS One 2019;14:e0214676.

7. Rosario PW. Subclinical Hypothyroidism. N Engl J Med 2017;377:1404.

8. Magri F, Chiovato L, Croce L, Rotondi M. Thyroid hormone therapy for subclinical hypothyroidism. Endocrine 2019;66:27-34.

9. Leonards CO, Schneider HJ, Liman TG, Fiebach JB, Endres M, Ebinger M. Thyroid-stimulating hormone, white matter hyperintensities, and functional outcome in acute ischemic stroke patients. Cerebrovasc Dis Extra 2014;4:61-8.

10. Zhang X, Xie Y, Ding C, Xiao J, Tang Y, Jiang X, Shan H, Lin Y, Zhu Y, Li C, Hu D, Ling Z, Xu G, Sheng L. Subclinical hypothyroidism and risk of cerebral small vessel disease: A hospital-based observational study. Clin Endocrinol (Oxf) 2017;87:581-6.

11. Schwarz S, Schwab S, Klinga K, Maser-Gluth C, Bettendorf $M$. Neuroendocrine changes in patients with acute space occupying ischaemic stroke. J Neurol Neurosurg Psychiatry 2003;74:725-7.

12. Peeters RP, Debaveye Y, Fliers E, Visser TJ. Changes within the thyroid axis during critical illness. Crit Care
Clin 2006;22:41-55, vi.

13. Sato S, Delcourt C, Heeley E, Arima H, Zhang S, AlShahi Salman R, Stapf C, Woo D, Flaherty ML, Vagal A, Levi C, Davies L, Wang J, Robinson T, Lavados PM, Lindley RI, Chalmers J, Anderson CS; INTERACT2 Investigators. Significance of Cerebral Small-Vessel Disease in Acute Intracerebral Hemorrhage. Stroke 2016;47:701-7.

14. Kumar M, Modi S, Rana P, Kumar P, Kanwar R, Sekhri T, D'souza M, Khushu S. Alteration in intrinsic and extrinsic functional connectivity of resting state networks associated with subclinical hypothyroid. J Neuroendocrinol 2018. [Epub ahead of print]. doi: 10.1111/jne.12587.

15. Ojala AK, Schalin-Jäntti C, Pitkälä KH, Tilvis RS, Strandberg TE. Serum thyroid-stimulating hormone and cognition in older people. Age Ageing 2016;45:155-7.

16. Zhang CE, Wong SM, Uiterwijk R, Backes WH, Jansen JFA, Jeukens CRLPN, van Oostenbrugge RJ, Staals J. Blood-brain barrier leakage in relation to white matter hyperintensity volume and cognition in small vessel disease and normal aging. Brain Imaging Behav 2019;13:389-95.

17. Xu X, Lau KK, Wong YK, Mak HKF, Hui ES. The effect of the total small vessel disease burden on the structural brain network. Sci Rep 2018;8:7442.

18. Dagre AG, Lekakis JP, Protogerou AD, Douridas GN, Papaioannou TG, Tryfonopoulos DJ, Papamichael CM, Alevizaki M. Abnormal endothelial function in female patients with hypothyroidism and borderline thyroid function. Int J Cardiol 2007;114:332-8.

19. Brown R, Benveniste H, Black SE, Charpak S, Dichgans M, Joutel A, Nedergaard M, Smith KJ, Zlokovic BV, Wardlaw JM. Understanding the role of the perivascular space in cerebral small vessel disease. Cardiovasc Res 2018;114:1462-73.

20. Duperron MG, Tzourio C, Sargurupremraj M, Mazoyer B, Soumaré A, Schilling S, Amouyel P, Chauhan G, Zhu YC, Debette S. Burden of Dilated Perivascular Spaces, an Emerging Marker of Cerebral Small Vessel Disease, Is Highly Heritable. Stroke 2018;49:282-7.

21. Mestre H, Tithof J, Du T, Song W, Peng W, Sweeney AM, Olveda G, Thomas JH, Nedergaard M, Kelley DH. Flow of cerebrospinal fluid is driven by arterial pulsations and is reduced in hypertension. Nat Commun 2018;9:4878.

22. Gutierrez J, DiTullio M, K Cheung YK, Alperin N, Bagci 
A, L Sacco R, B Wright C, Sv Elkind M, Rundek T.

Brain arterial dilatation modifies the association between extracranial pulsatile hemodynamics and brain perivascular spaces: the Northern Manhattan Study. Hypertens Res

Cite this article as: Chu M, Cai Y, Zhong J, Qian Y, Cen Y, Dou M, Chen G, Sun B, Lu X. Subclinical hypothyroidism is associated with basal ganglia enlarged perivascular spaces and overall cerebral small vessel disease load. Quant Imaging Med Surg 2022;12(2):1475-1483. doi: 10.21037/qims-21-190
2019;42:1019-28.

23. Marmarou A, Takagi H, Shulman K. Biomechanics of brain edema and effects on local cerebral blood flow. Adv Neurol 1980;28:345-58. 


\section{Supplementary}

Table S1 Risk factors of increasing of $\mathrm{a} / \mathrm{b}$ and the reducing of $\mathrm{c}$-line distance. Ordinal regression was performed to investigate the association of subclinical hypothyroidism with brain atrophy. $\mathrm{a} / \mathrm{b}$ and c-line distance were categorized as quartiles. More increasing of a/b or reducing of c-line distance indicated severe brain atrophy. After classified by quartiles, neither the increasing of $\mathrm{a} / \mathrm{b}$ nor the reducing of $\mathrm{c}$-line distance was associated with subclinical hypothyroidism. Age, male gender, and EPVS were risk factors of increasing of $\mathrm{a} / \mathrm{b}$ and the reducing of c-line distance

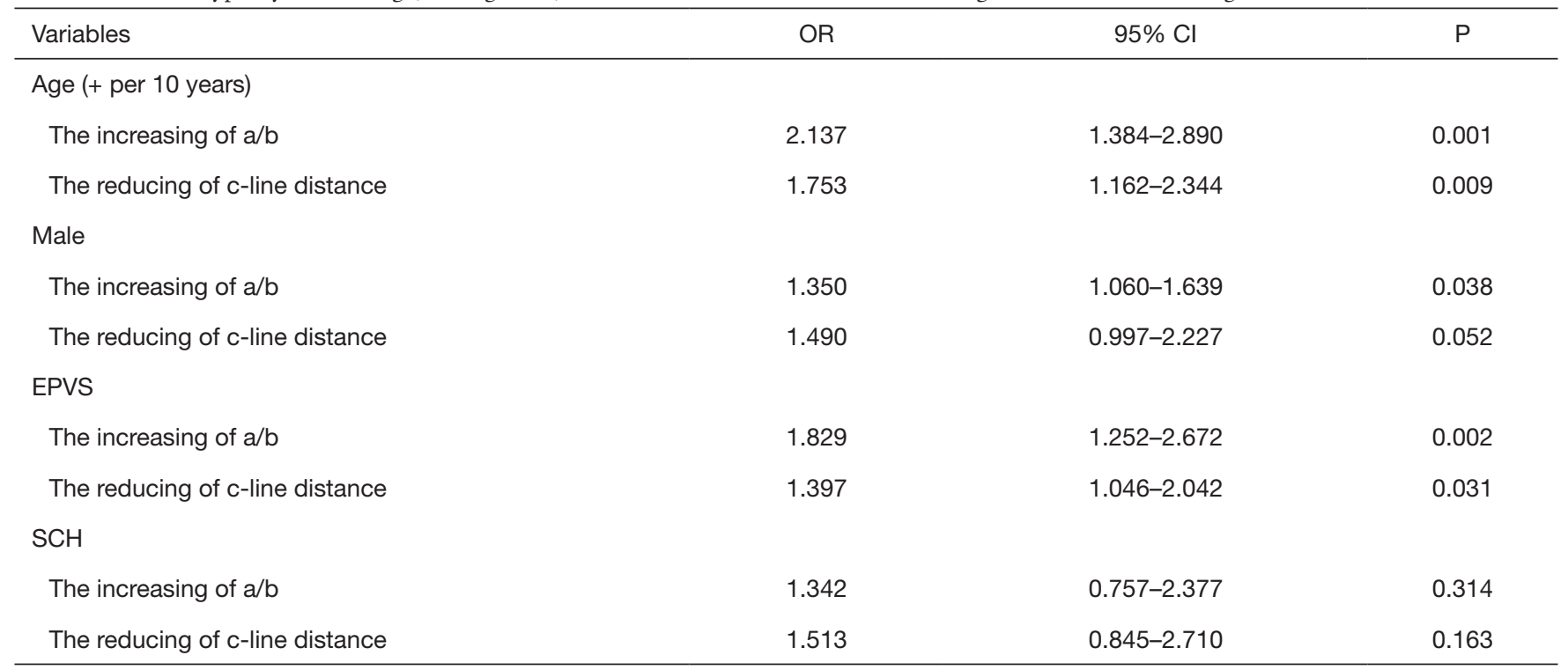

"a" and "b" were defined as the maximum measurement from the central line to lateral margin of anterior horn of lateral ventricle and the maximum measurement from the central line to lateral margin of frontal lobe at the axial level of the basal ganglia, respectively. "c-line" was defined as the maximum measurement from the central line to lateral margin of insular cortex at the same level. EPVS, enlarged perivascular spaces; $\mathrm{SCH}$, subclinical hypothyroidism; OR, odds ratio; $\mathrm{Cl}$, confidence interval. 